\title{
Surgical management of Ossifying Fibroma of the mandible with inferior alveolar nerve involvement
}

\author{
Yadegari $\mathbf{A}^{\mathbf{1}}$, SeyyedKhamesi $\mathbf{S}^{2}$, Aminian $_{\mathbf{M}^{3}}$ \\ ${ }^{1}$ Assistant professor and head, Oral and maxillofacial surgery Dept, Isfahan(Khorasgan) branch, Islamic Azad University, Isfahan, Iran. \\ 2 Post-G raduate student, O ral and maxillofacial surgery Dept, Isfahan(K horasgan) branch, Islamic Azad U niversity, Isfahan, Iran. \\ ${ }^{3}$ P ost-G raduate student,D ental student's Research Committee, D ental faculty, I sfahan U niversity of M edical Science, I sfahan, Iran
}

\begin{tabular}{l}
\hline ARTICLE INFO \\
\hline Article Type \\
Case Report \\
Article History \\
Received: Jan 2017 \\
Accepted: F eb 2017 \\
eP ublished: Apr 2017 \\
\hline Keywords: \\
Ossifying Fibroma, \\
Maxillofacial Surgery, \\
Mandible,
\end{tabular}

\begin{abstract}
Background and Aim: Ossifying fibroma (OF) is a benign fibro-osseous lesion, which is usually diagnosed between the ages of 20 and 40 years. The female to male ratio is 5:1, with affinity for posterior mandibular region. The aim of the present report is to discuss a case of OF and its clinical, radiological and pathological characteristics and the related surgical approach.

Case presentation: A left mandibular impacted premolar with an associated lesion was incidentally found on a radiograph of a 27 -year-old woman. The patient did not report any clinical symptoms. Inferior alveolar nerve involvement by the lesion was obvious in radiographic study. The tooth was extracted and the associated lesion was enucleated under general anesthesia through step-by-step surgery and after releasing the inferior alveolar nerve.

Conclusion: Surgical treatment of Ossifying fibroma consists of enucleation and curettage in small and well-defined lesions, while larger lesions are usually resected. Prognosis is good with a low recurrence rate even with enucleation and after longterm follow-up.
\end{abstract}

\footnotetext{
Please cite this paper as: Yadegari A, Seyyedkhamesi S, Aminian M. Surgical management of Ossifying Fibroma of the mandible with inferior alveolar nerve involvement. J Res Dentomaxillofac Sci. 2017;2(2):44-50
}

\footnotetext{
*Corresponding author: 


\section{Introduction:}

Ossifying Fibroma (OF) is a benign fibroosseous neoplasm with progressive growth that shows expansion and well-defined borders on radiographs.

Histologically, OF is comprised of bone or cementum-like substances in a fibrous connective tissue stroma. ${ }^{(1)}$

OF accounts for 2 to $4 \%$ of jaw lesions (similar to Fibrous Dysplasia). The age of occurrence varies from 8 to 53 years, and most of the patients are in the second to the fourth decades of life. The female to male ratio is $1: 5 .^{(1,2)}$

OF has two distinctive characteristics: slow growth and distinct margins from the surrounding bone. ${ }^{(3)}$

OF develops almost exclusively in facial bones. The incidence rate is 62 to $89 \%$ in the mandible, ${ }^{(4)}$ mostly involving the premolar and molar areas. ${ }^{(2)}$ Maxillary lesions are reported both in the anterior and posterior regions. ${ }^{(1)}$ Other facial areas such as periorbital, frontal, ethmoid, sphenoid and temporal bones are common areas for this lesion. ${ }^{(3)}$ Sinuses and nasal cavity are rarely involved. ${ }^{(5)}$

Some OF lesions can grow excessively and may jeopardize aesthetics and function. ${ }^{(3)} \mathrm{OF}$ occurring at an early age is called Juvenile Aggressive Ossifying Fibroma, which is an aggressive and hypervascular lesion. ${ }^{(4)}$

\section{Case presentation:}

A left mandibular impacted premolar with an associated lesion was incidentally found on a radiograph of a 27-year-old woman. The patient did not report any clinical symptoms.

In review of the patient's medical history, severe neurological disorders and stress were reported. She had visited a psychologist two months previously. The patient was consuming Chlordiazepoxide $5 \mathrm{mg}$, Sertraline $100 \mathrm{mg}$, and Depakin $500 \mathrm{mg}$.

There was no history of addiction or food and medicinal allergies. The patient's family history revealed that her parents were suffering from diabetes and hypertension.

In checking the laboratory examination, Hemoglobin $(=10.5 \mathrm{~g} / \mathrm{dl}), \mathrm{MCH}(=24.2 \mathrm{pg})$ and
MCHC (=28.9 g/dl) were slightly lower than normal while other factors were normal.

In extraoral examination (Figure 1, A), no specific pain, swelling, numbness, tenderness, or asymmetry were noted. The patient had no history of trauma in the area.

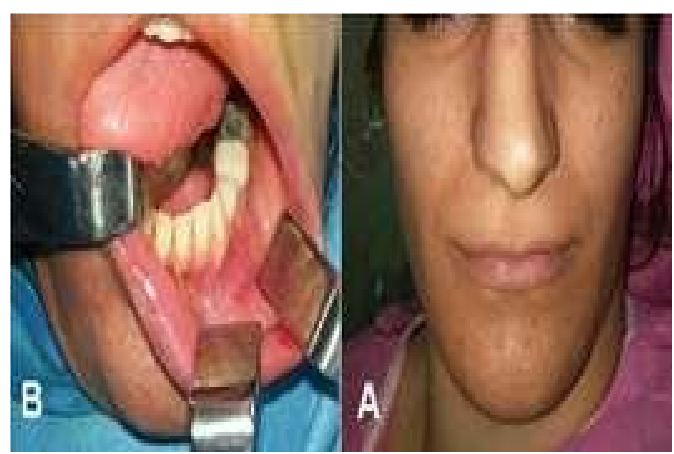

Figure 1- A. Extraoral view (no asymmetry) B. Intraoral view

In intraoral examination (Figure 1, B), all teeth were present except wisdom teeth on both sides and the left mandibular second premolar The left mandibular first molar was crowned and the left mandibular second molar had an extensive filling. Other teeth were vital and without decay or extensive filling. Mild swelling was detected in the buccal and lingual sides of the left mandibular premolar area; however, buccal and lingual plates were bony hard upon palpation and there was no evidence of perforation. Vestibular mucosa of the left mandibular second premolar area showed normal color with no tenderness or any other signs.

In evaluation of panoramic and $\mathrm{CBCT}$ views (Figure 2), a mixed radiopaque-radiolucent lesion with distinct margins was observed at the apical part of the left mandibular impacted second premolar which was attached to the root. The maximum dimensions of the lesion were estimated by CBCT as $17.4 \mathrm{~mm}$ mesio-distally and 11.6 mm bucco-lingually.

In CBCT views, mild expansion was detected in buccal and lingual cortical borders. Buccal cortex was thinned, and perforation was detectable in a single point. The inferior alveolar nerve canal was displaced lingually and had a close contact with the second premolar's root.

The patient went under general anesthesia. Local anesthetic agent (lidocaine 2\% containing 


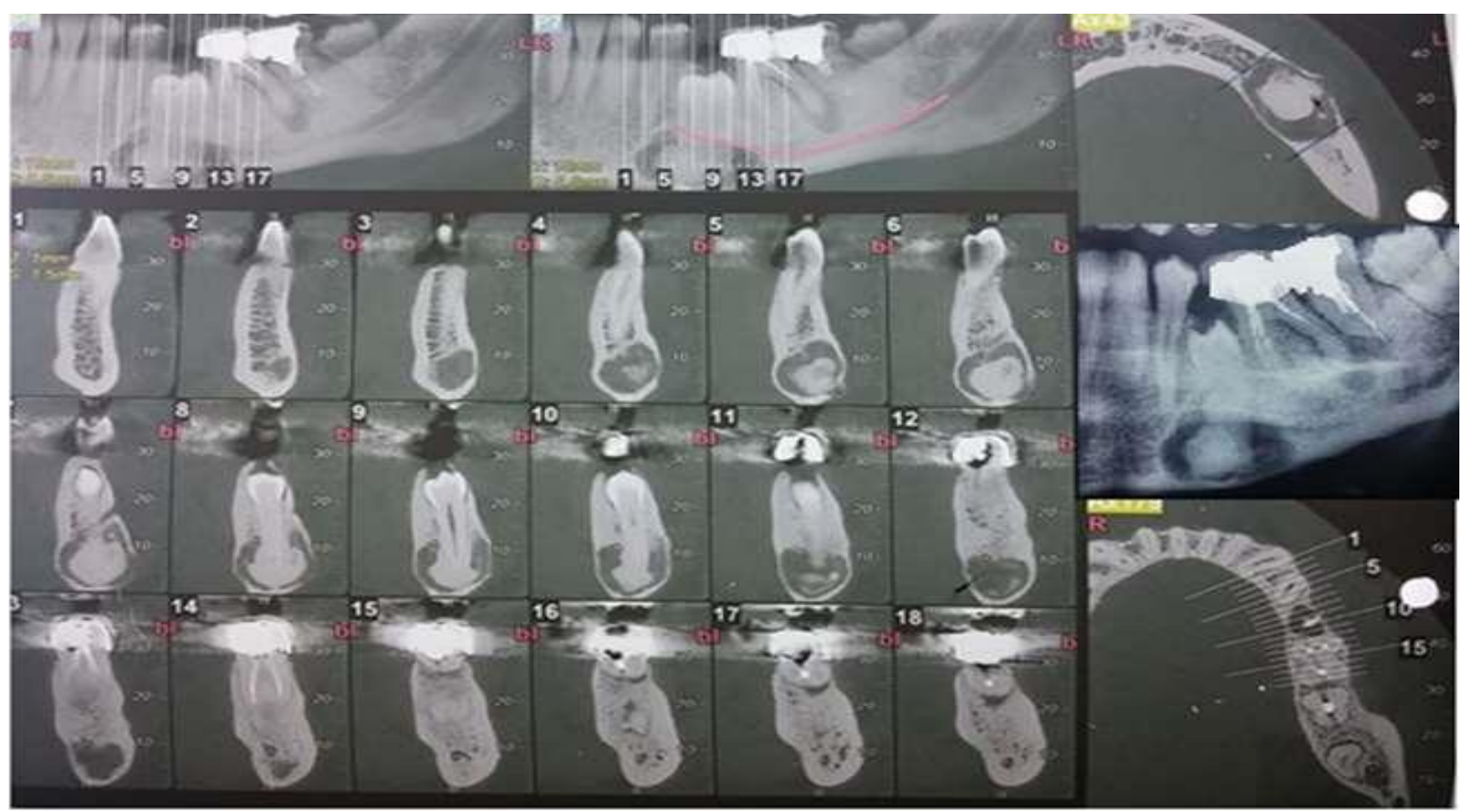

Figure 2: CBCT cross-sectional views. Sections 8 to 11 show the lingual proximity of the inferior alveolar nerve canal with the root of the impacted second premolar and also close contact with the lesion. Thinning and perforation of the buccal cortex are seen in sections 6 to 8 and also in the axial view.

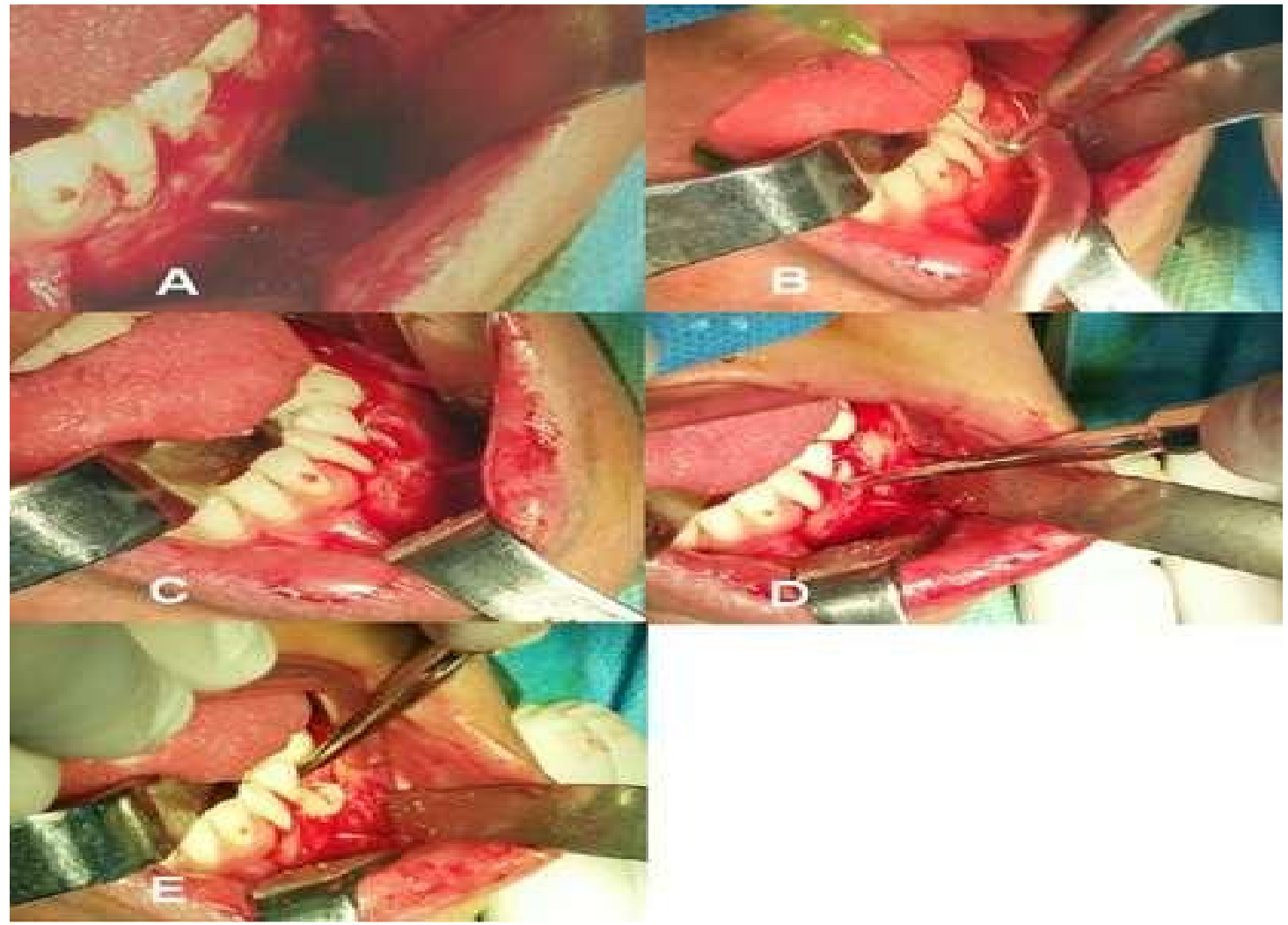

Figure 3- A. The mucosa is elevated subperiosteally, and cusp tip of the impacted second premolar and the mental nerve are visible. $B$ and $C$. The cortical bone on the coronal area of the second premolar was removed by a round bur mounted on a surgical handpiece and the dental crown became fully visible. $D$ and $E$. The coronal portion was separated from the root by the use of a surgical fissure bur and was extracted by using a direct elevator. 


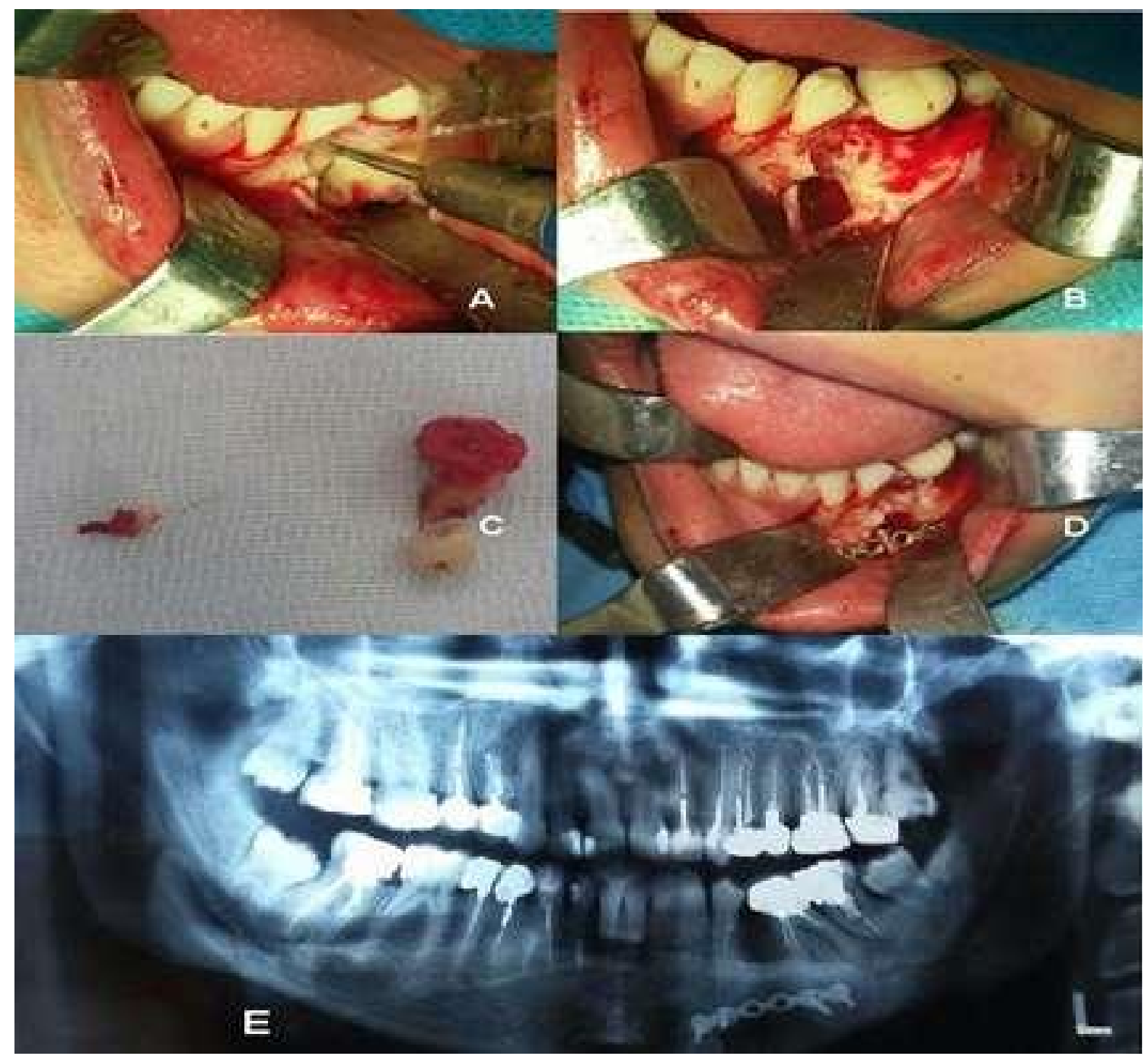

Figure 4: A. After cautiously displacing the mental nerve, the buccal and apical bony regions covering the radicular area and the lesion were removed. $B$ and $C$. After full enucleation of the lesion, curettage was performed on the bony walls by using a large round bur. D: The plate was carefully passed under the inferior alveolar nerve and was fixed to both sides of the remained defect. The bone cavity was filled with Surgicel ${ }^{\circledR}$ as cavity filler. E:

epinephrine) was injected in the buccal vestibule of the left side of the mandible in order to elevate the tissue and decrease local bleeding during surgery. Seven minutes after the injection, the vestibular incision was extended from the left second molar area to the central incisor area encompassing two layers (mucosal and muscular).

Figure 3 shows exposure of the tooth, crown-root sectioning and extraction process. Since the periapical lesion was tightly connected with the root, it was impossible to extract the root by using a direct elevator; there- fore, after

cautiously retracting the mental nerve, the buccal bone covering the root and the lesion was removed.

Since the inferior alveolar nerve was located inside the buccal and apical aspects of the periapical lesion, a part of the nerve which was traced inside the lesion was carefully released by using a fissure bur. The extracted root and the associated enucleated lesion were sent for histopathology examination. (Figure 4. A-C)

The mandibular bone seemed fragile after enucleation; therefore, a 6-hole plate with four no. 
7 screws (the mini plate system) was placed in the area to reinforce the mandibular bone. (Figure 4, D) Both mucosal and muscular layers in the area were sutured. The follow-up panoramic image was taken 2 weeks (Figure 4. E), and also 12 months (Figure 5) after the surgery and no symptoms of infection were reported by the patient. (The patient was asked to treat the left mandibular first and second molars and left maxillary third molar.)

\section{Histopathology Examination:}

The sample included several creamy-white soft tissue pieces with the dimensions of $3 \times 1 \times 2$ $\mathrm{cm}$ and two hard tissue pieces with the dimensions of $0.7 \times 0.3 \times 1 \mathrm{~cm}$ with firm consistency, which were associated with a single-rooted tooth. (Figure 6. A)

In microscopic evaluation and histopathology examination, a fibrocellular connective tissue stroma with calcified elements including basophilic spherical-shaped areas associated with irregular calcified structures containing osteoblasts and osteoid foci were observed. The connective tissue cells mostly contained large and fairly elongated nuclei; therefore, there were no signs of malignant cellular transformation or atypism. (Figure 6. B-C) After considering the histopathology report, clinical information and radiographic characteristics, the final diagnosis of Ossifying Fibroma was made.

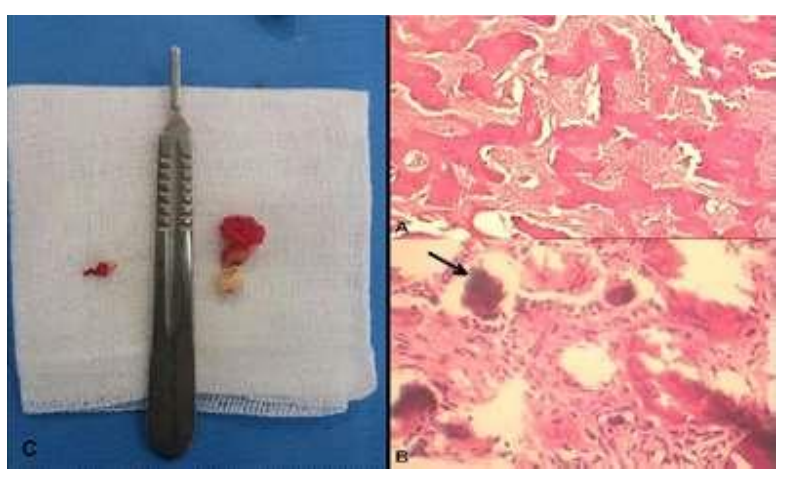

Figure 6-A. The sample included several soft and hard tissue pieces B. Fibrocellular connective tissue and calcified structures. $C$. The basophilic spherical-shaped areas.

\section{Discussion:}

Cemento-Ossifying Fibroma (COF) is classified as an osteogenic tumor. ${ }^{(5,6)}$ In 1992, world health organization (WHO) used the term COF in classifying the odontogenic tumors. This term had not been used for a long time. The reason of this naming was that these lesions were connected to teeth, and also cementum-like materials were present even at the remote areas around the tooth. This nomenclature was changed in 2005 and currently, all types of COF are referred to as Ossifying Fibroma (OF). ${ }^{(1,7)}$ Ossifying Fibroma is a benign fibro-osseous neoplasm with progressive growth which causes expansion and has well-defined borders on radiographs.

Histologically, OF is comprised of bone or cementum-like substances in a fibrous connective

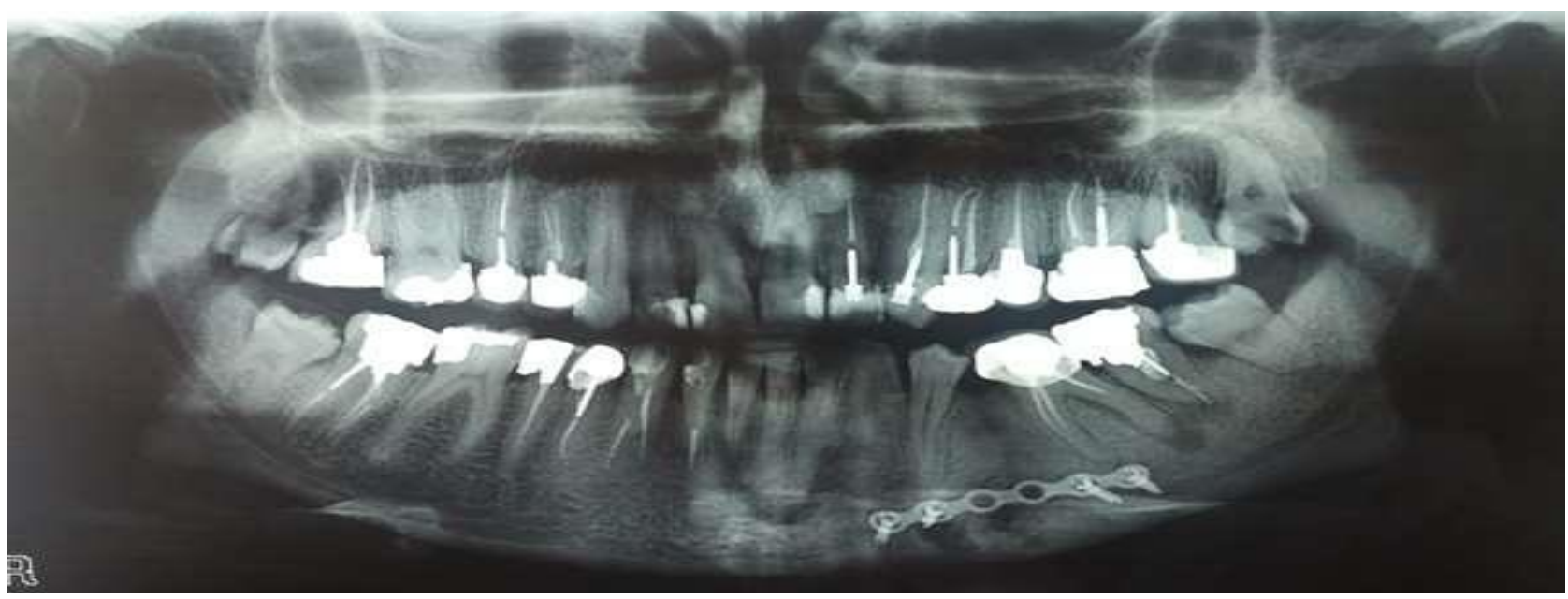

Figure 5- Panoramic radiograph taken 12 months after the surgery. 
tissue stroma. ${ }^{(1)}$

The clinical manifestation of OF may vary from a gradual growth to an invasivebehavior. (8) Although inflammation of buccal and lingual cortical plates is common, buccal pain and perforation are rare. In general, there are no specific clinical or microscopic characteristics to help predict the behavior of this tumor, ${ }^{(1)}$ and the majority of these lesions are discovered incidentally during routine dental examinations. The rapid growth of Juvenile Ossifying Fibroma may cause jaw deformity. ${ }^{(8)}$

In radiographic views, most of these lesions show distinct margins and spherical or oval shapes with cortication or sclerosis. ${ }^{(1)}$ The tumor-like behavior of this lesion causes growth in the medullary bone and consequently a relative equal and concentric expansion in all directions. This characteristic causes thinning and displacement of the cortical borders with no perforation. ${ }^{(8)}$ Mandibular lesions are always situated above the inferior alveolar nerve canal and displace the canal downwards. Accordingly, dental displacement may occur and root resorption is observable in $44 \%$ of the cases. ${ }^{(1)}$

OF shows a radiolucent, mixed, and totally radiopaque maturation process in radiographs. (3) The pattern of the internal structure depends on the amount and shape of calcified and bony substances. ${ }^{(7)}$ The lesion is usually hard to identify and differentiate before producing calcification. ${ }^{(4)}$ In radiopaque lesions, a thin radiolucent rim always separates the lesion from the surrounding bone and actually represents the fibrous capsule around the tumor. ${ }^{(8)}$

The majority of OFs are separated from the normal surrounding bone by a thin layer of fibrous tissue that makes it possible to perform an easy surgery and completely remove the lesion. $\mathrm{OF}$ is made of hyper-cellular fibroblastic stroma with collagen fibers, blood vessels and varying amounts of mineral substances. Multinucleated giant cells which are mostly osteoclasts can be seen in some areas. ${ }^{(1)}$ The most common calcification pattern is small osteoid trabeculae surrounded by osteoblasts. Under polarized light, the primary tumors contain woven bone, while mature lesions contain trabeculae of lamellar bone. Usually, more than one pattern is observed in a single lesion which helps distinguish OF from Fibrous Dysplasia (FD). ${ }^{(1)}$
Histological characteristics of fibro-osseous lesions have a significant overlap with each other; accordingly, a correct diagnosis requires simultaneous histological, clinical and radiographic evaluations. ${ }^{(3)}$

In general, differential diagnoses of $\mathrm{OF}$ include round radiolucent lesions containing a series of radio-opacities including: Fibrous Dysplasia (FD), Periapical Osseous Dysplasia (POD), Calcifying Odontogenic Cyst (COC or Gorlin cyst), Calcifying Epithelial Odontogenic Tumor (CEOT or Pindborg tumor), and Osteosarcoma or Chondrosarcoma. ${ }^{(4)}$ Differentiating OF from FD is the main challenge in differential diagnosis. The most helpful characteristic is the distinct margins of OF in radiography and consequently, easy separation from the normal bone in surgery. ${ }^{(3)}$ The ground glass view is a characteristic of FD which is not seen in OF ${ }^{(4)}$ Histologically, FD only contains woven bone (immature) and unlike OF, there is no evidence of an osteoblastic rim around the bone. It is believed that the presence of mature lamellar bone is a characteristic of $\mathrm{OF}^{(3)}$

$\mathrm{OF}$ is histopathologically indistinguishable from POD. ${ }^{(6)}$ The differentiation is usually possible with the help of radiographic views. Unlike OF, POD is often multifocal. Other characteristics of POD which are different from that of OF are as follows: presence of Simple Bone Cysts (SBC), wide sclerotic border, and waveform expansion. OF shows a more tumor-like behavior with displacing the tooth and concentric expansion. ${ }^{8}$

Differentiating OF from Gorlin Cyst and Pindborg tumor is difficult with radiography. The final diagnosis is made based on the histologic view. There is a strong connection between Pindborg tumor and the impacted tooth. ${ }^{(4)}$

Sarcomas show characteristics that suggest malignancy, such as cortical bone destruction, invasion to the surrounding soft tissue, and destruction along the periodontal ligament space; ${ }^{(9)}$ however, OF has a welldefined border. ${ }^{(4)}$

Treatment of OF consists of enucleation and curettage in small and well-defined lesions, while larger lesions are usually resected. The prognosis is good ${ }^{(9)}$ with a low 
recurrence rate even with enucleation and after long-term follow-up. ${ }^{(1)}$

\section{Conflicts of Interest:}

the authors of this manuscript certify that they have no conflicts of interest regarding this research.

\section{References:}

1. Mohsenifar Z, Nouhi SA, Mashhadi Abbas F, Farhadi S, Abedin B. Ossifying fibroma of the ethmoid sinus: Report of a rare case and review of literature. J Res Med Sci. 2011;16(6):841-7.

2. Shimamoto H, Kishino M, Okura M, Chindasombatjaroen J, Kakimoto N, Murakami S, et al. Radiographic features of a patient with both cemento-ossifying fibroma and keratocystic odontogenic tumor in the mandible: a case report and review of literature. Oral surg Oral Med Oral Pathol Oral Radiol 2011;112(6):798-802.

3. Triantafillidou K, Venetis G, Karakinaris G, Iordanidis F. Ossifying fibroma of the jaws: a clinical study of 14 cases and review of the literature. Oral surg Oral Med Oral Pathol Oral Radiol 2012;114(2):193-9.

4. Hekmatnia A, Ghazavi A, Saboori M, Mahzouni P, Tayari N, Hekmatnia F. A case report of cemento-ossifying fibroma presenting as a mass of the ethmoid sinus. J Res Med Sci 2011;16(2):224-8.

5. Trijolet JP, Parmentier J, Sury F, Goga D, Mejean N, Laure B. Cemento-ossifying fibroma of the mandible. Eur Ann Otorhinolaryngol Head Neck Dis 2011;128(1):30-3.

6. Ram R, Singhal A, Singhal P. Cemento-ossifying fibroma. Contemp clin dent 2012;3(1):83-5.

7. Mohan RP, Verma S, Singh U, Agarwal N. Cementifying fibroma. BMJ Case Rep 2013;8(1): 13-5.

8. Urs AB, Kumar P, Arora S, Augustine J. Clinicopathologic and radiologic correlation of ossifying fibroma and juvenile ossifying fibroma-an institutional study of 22 cases. Ann diagn pathol 2013;17(2):198-203.

9.Gerlach RC, Dixon DR, Goksel T, Castle JT, Henry WA. Case presentation of florid cementoosseous dysplasia with concomitant cementoossifying fibroma discovered during implant explantation. Oral surg Oral Med Oral Pathol Oral Radiol 2013;115(3):44-52. 